\title{
Widening operators for powerset domains
}

\author{
Roberto Bagnara · Patricia M. Hill • Enea Zaffanella
}

Published online: 27 February 2007

(C) Springer-Verlag 2007

\section{Erratum to: Int J Softw Tools Technol Transfer 8(4/5): 449-466 \\ DOI 10.1007/s10009-005-0215-8}

In the printed version of this article unfortunately all figures are incomplete. Please find the correct version of all figures enclosed and in the online version of the original article.

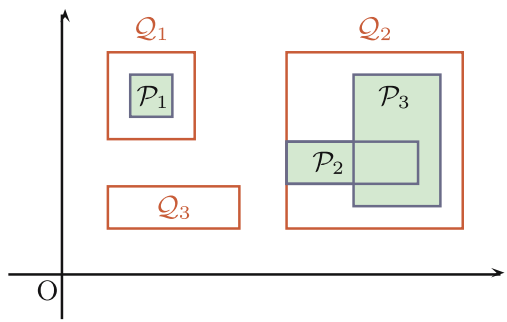

Fig. 1 The collection of $\mathcal{Q}_{i}$ approximates the collection of $\mathcal{P}_{i}$

The online version of the original article can be found at http://dx.doi.org/10.1007/s10009-005-0215-8.

R. Bagnara $(\bowtie) \cdot$ E. Zaffanella

Department of Mathematics,

University of Parma, Parma, Italy

e-mail: bagnara@cs.unipr.it

E. Zaffanella

e-mail: zaffanella@cs.unipr.it

P. M. Hill

School of Computing,

University of Leeds, Leeds, UK

e-mail: hill@comp.leeds.ac.uk
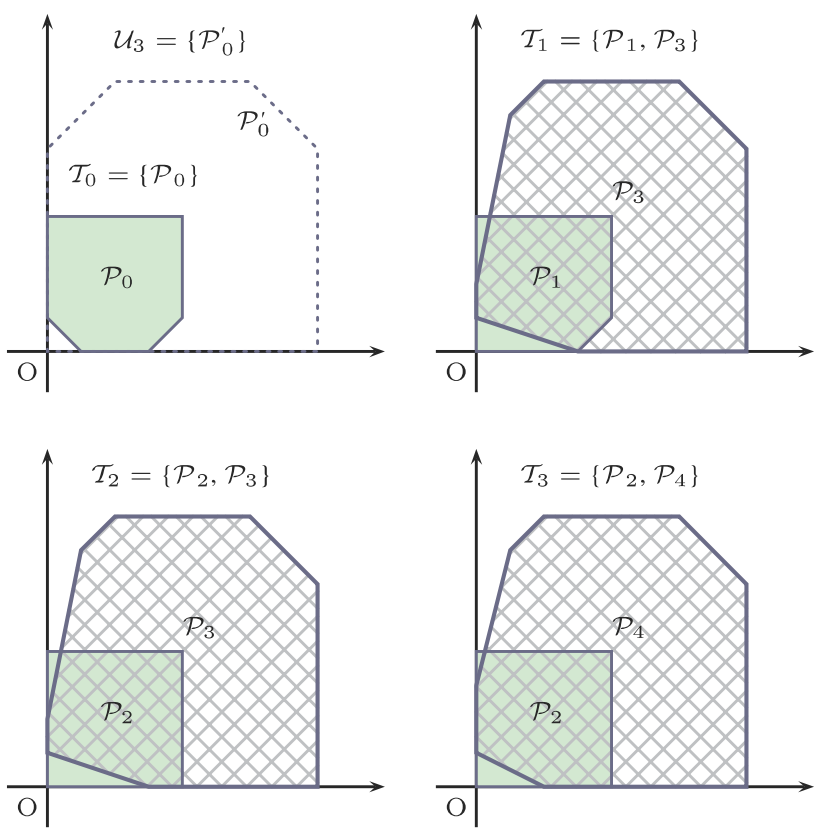

Fig. 2 The iterates $\mathcal{T}_{0}, \mathcal{T}_{1}, \mathcal{T}_{2}, \mathcal{T}_{3}$ and $\mathcal{U}_{3}$ in Example 3

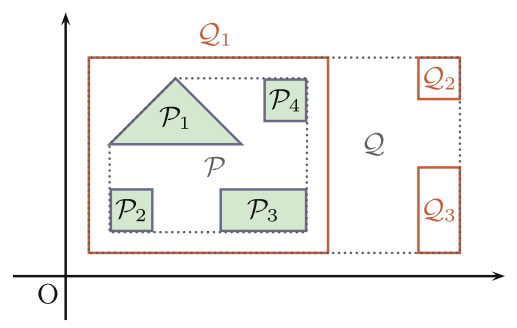

Fig. 3 The lgo relation on collections of polyhedra 


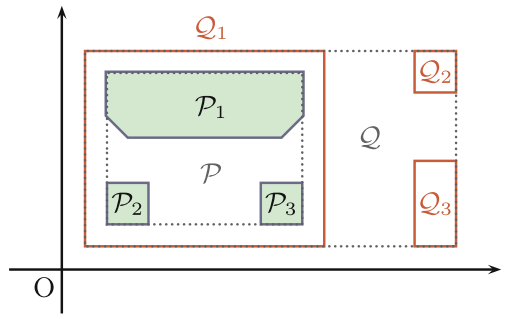

Fig. 4 The lgo relation on collections of polyhedra
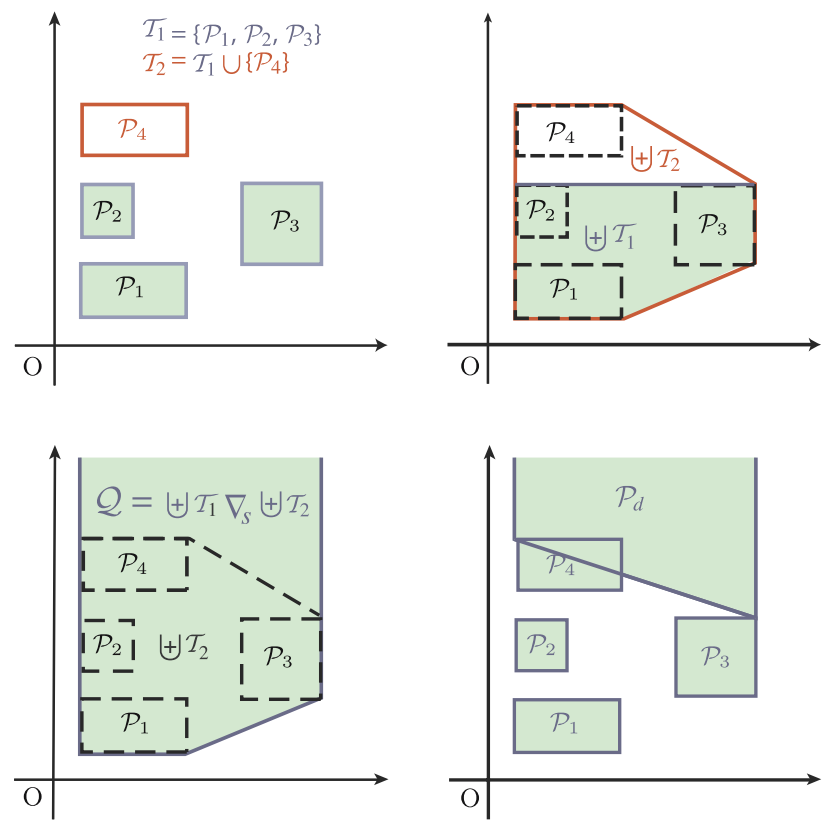

Fig. 5 The second case of the certificate-based widening
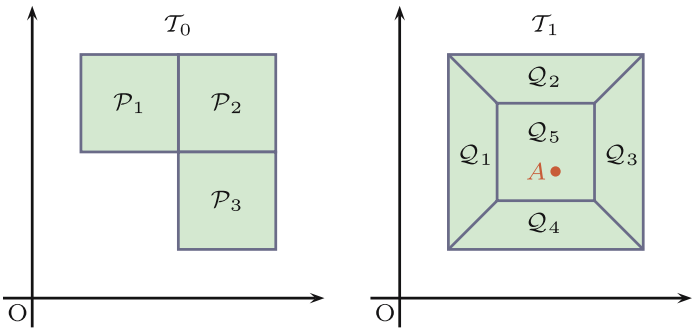

Fig. 6 Merging polyhedra according to ' $₫$ '
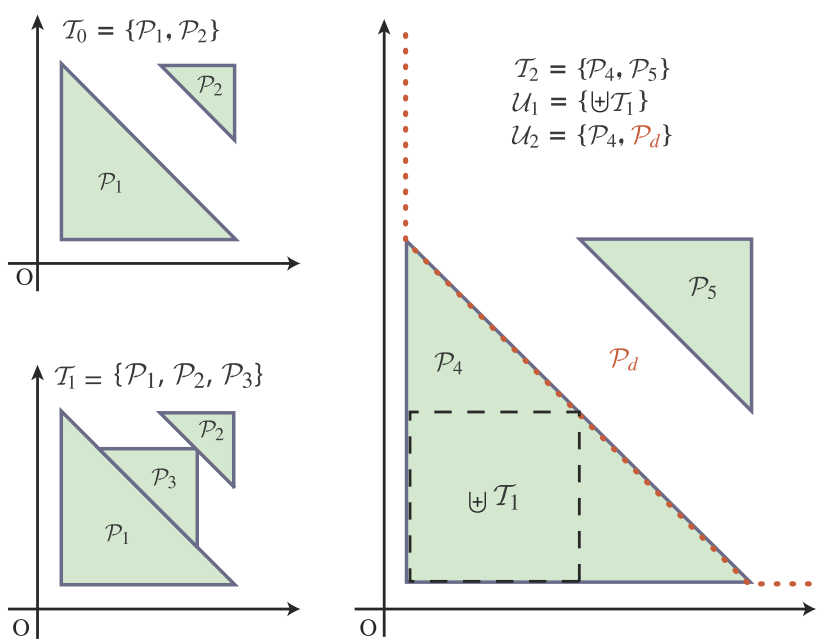

Fig. A.1 The condition $\# \mathcal{U}_{1}>1$ is needed to obtain a proper widening 